\title{
Simplified Equations for Computing Science Orbits Around Planetary Satellites
}

\author{
Martin Lara* \\ Real Observatorio de la Armada, 11110 San Fernando, Spain
}

DOI: $10.2514 / 1.31107$

\begin{abstract}
An analytical theory for spacecraft motion close to synchronously orbiting and rotating planetary satellites is provided. The ratio rotation rate of the satellite mean motion of the orbiter is the small parameter of the theory. A double-averaging over the mean anomaly and the argument of the node reduces the problem to one degree of freedom in the eccentricity and the argument of the periapsis. The theory is based on the Lie-Deprit perturbation method, which permits recovering the short- and long-period terms through explicit transformations. An application to the computation of (unstable) science orbits is presented, a case in which the transformation equations admit dramatic simplifications.
\end{abstract}

\section{Introduction}

$\mathbf{C}$ ONCERNING the search for life on the solar system, planetary satellites are pointed to as major targets by the scientific community. Mapping missions require low-eccentricity and highinclination orbits close to the central body, a kind of orbit that is known to be unstable for planetary satellites. The main perturbations in the motion around planetary satellites arise from the nonsphericity of the central body and the planetary perturbation. Many natural satellites orbit synchronously the planet. Therefore, Kozai's [1] model for a lunar orbiter is well-adapted for studying the dynamics around planetary satellites [2].

With Kozai [1], we consider the mean gravitational field of a synchronously rotating and orbiting moon and take into account the perturbations of the third body in the Hill problem approximation. Commonly, solar system bodies show latitudinal asymmetry and we left open our theory to the inclusion of this effect, which can play an important role $[3,4]$. A double average over the mean anomaly and the argument of the node reduces the problem to one degree of freedom (DOF) in the eccentricity and the argument of the periapsis. The phase space of the integrable reduced problem is made of closed curves and equilibria, the latter corresponding to trajectories that are known as frozen orbits. Using perturbation techniques based on Lie transforms [5], the short- and long-period terms are recovered through explicit transformations.

An immediate application of our theory is found for the computation of long-lifetime science orbits. Although loweccentricity frozen orbits are known to exist for all the range of inclinations, the third-body perturbations produce unstable behavior on high-inclination orbits in a wide range of inclinations centered about polar orbits [6]. These instabilities result in relatively short lifetimes, on the order of one month [3] . As proposed in [7], initial conditions can be chosen on the stable manifold of the desired frozen orbit. Then the explicit equations of the transformations provide initial conditions of the nonaveraged problem corresponding to nominal orbits that, in average, follow the stable-unstable manifold of unstable frozen orbits, increasing the lifetime of a science mission about a planetary satellite. An advantage of the Lie transforms approach is that it avoids the linearization procedure required by

Presented as Paper 2007-221 at the AAS/AIAA Space Flight Mechanics Meeting, Sedona, AZ, 28 January-1 February 2007; received 18 March 2007; revision received 3 August 2007; accepted for publication 3 August 2007. Copyright $(\odot 2007$ by the American Institute of Aeronautics and Astronautics, Inc. All rights reserved. Copies of this paper may be made for personal or internal use, on condition that the copier pay the $\$ 10.00$ per-copy fee to the Copyright Clearance Center, Inc., 222 Rosewood Drive, Danvers, MA 01923; include the code 0731-5090/08 \$10.00 in correspondence with the CCC.

${ }^{*}$ Commander, Ephemerides Section. Member AIAA. other methods to recover the long- and short-period effects missed in the averaging [7].

This paper is the last step in an effort to build an analytical theory for mission analysis of science orbits around planetary satellites. The initial studies of the double-averaged problem $[6,8]$ were followed by a first attempt to recover the original dynamics through explicit transformation equations [9]. However, lacking information about the short-period terms, the analytical solutions of [9] commonly require a numerical refinement of the orbital elements. The transformations for recovering both the short- and long-period dynamics were used in [10] for the first time, but being affected by negative powers of the eccentricity, their application was limited to moderate-eccentricity orbits. Transformation equations in nonsingular elements are available now up to the second order. Furthermore, for the low eccentricities and high inclinations required for science orbits, the whole sequence of transformations may be replaced by the single set of simplified transformation equations provided in this paper.

\section{Dynamic Model and Perturbation Method}

When considering a synchronously orbiting planetary satellite for which the equator coincides with its orbital plane, the problem is invariant in a rotating frame of reference with the origin at the center of mass of the satellite. Using Hamiltonian formulation, we write

$$
\mathcal{H}=(1 / 2)(\boldsymbol{X} \cdot \boldsymbol{X})-\boldsymbol{\omega} \cdot(\boldsymbol{x} \times \boldsymbol{X})-(\mu / r)-R(\boldsymbol{x})
$$

where $\boldsymbol{x}=(x, y, z)$ is the position vector of the orbiter, $r=\|\boldsymbol{x}\|$, $\boldsymbol{X}=(X, Y, Z)$ is the vector of conjugated momenta (velocity in the inertial frame), $\omega$ is the angular velocity of the satellite around the planet, $\mu$ is the satellite's gravitational parameter, and the perturbing function $R$ includes the third-body and nonsphericity perturbations. In projective coordinates $r(u=x / r, v=y / r$, and $w=z / r)$, the perturbing function is

$$
R=\omega^{2} r^{2}\left(3 u^{2}-1\right) / 2+(\mu / r) N(r, u, v, w)
$$

with $\omega=\|\omega\|$. For the nondimensional part $N$ of the potential, we take

$$
\begin{aligned}
N & =(\alpha / r)^{2}\left[J_{2}\left(1-3 w^{2}\right) / 2+3 C_{2,2}\left(u^{2}-v^{2}\right)\right] \\
& +J_{3}(\alpha / r)^{3} w\left(3-5 w^{2}\right) / 2
\end{aligned}
$$

where the scaling factor $\alpha$ is the equatorial radius of the planetary moon, and $J_{2}, C_{2,2}$, and $J_{3}$ are the oblateness, ellipticity, and latitudinal asymmetry coefficients, respectively. The projective coordinates are expressed in polar nodal variables: 


$$
\begin{gathered}
u=\cos h \cos \theta-\cos I \sin h \sin \theta \\
v=\sin h \cos \theta+\cos I \cos h \sin \theta, \quad w=\sin I \sin \theta
\end{gathered}
$$

where $h$ is the argument of the node in the rotating frame, $I$ is inclination, $\theta=f+g, g$ is the argument of the periapsis, and $f$ is the true anomaly.

The Hamiltonian equation (1) is expressed as a perturbed twobody problem:

$$
\mathcal{H}=\mathcal{H}_{0}+\epsilon \mathcal{H}_{1}+\left(\epsilon^{2} / 2\right) \mathcal{H}_{2}+\left(\epsilon^{3} / 6\right) \mathcal{H}_{3}
$$

where the formal parameter $\epsilon$ manifests the order of each perturbation. The zero order is the Keplerian:

$$
\mathcal{H}_{0}=-\mu /(2 a)
$$

The first-order perturbation corresponds to the Coriolis force:

$$
\begin{aligned}
\mathcal{H}_{1} & =-\omega\|\boldsymbol{x} \times \boldsymbol{X}\| \cos I=-\omega \sqrt{\mu a\left(1-e^{2}\right)} \cos I \\
& =-\frac{\mu}{2 a}\left(\frac{\omega}{n}\right)\left(2 \sqrt{1-e^{2}} \cos I\right)
\end{aligned}
$$

where the elements $a$ and $e$ are the instantaneous semimajor axis and eccentricity, respectively, and $n=\sqrt{\mu / a^{3}}$ is the instantaneous mean motion of the orbiter. The effects of the oblateness and ellipticity of the satellite and the planetary perturbation remain at second order in $\omega / n$ :

$$
\begin{aligned}
\mathcal{H}_{2} & =-\frac{\mu}{2 a}\left(\frac{\omega}{n}\right)^{2} \frac{r^{2}}{a^{2}}\left\{\left(\frac{1}{2}+\beta^{2} \frac{a^{5}}{r^{5}}\right)\left(2-3 s^{2}+3 s^{2} \cos 2 \theta\right)\right. \\
& +\left(\frac{3}{4}+\frac{9 \beta^{2}}{10} \frac{a^{5}}{r^{5}}\right)\left[2 s^{2} \cos 2 h+(1-c)^{2} \cos (2 \theta-2 h)\right. \\
& \left.\left.+(1+c)^{2} \cos (2 \theta+2 h)\right]\right\}
\end{aligned}
$$

where we set $C_{2,2}=(3 / 10) J_{2}$ from equilibrium theory [11], and the function

$$
\beta=\frac{J_{2}^{1 / 2}}{(\omega / n)}(\alpha / a)
$$

expresses the relative importance of the oblateness and third-body perturbations. For brevity, we use the notations $c \equiv \cos I$ and $s \equiv \sin I$.

Despite $J_{3}$ vanishing in equilibrium theory, we consider a possible latitudinal asymmetry of the satellite for which the effect is of third order in $\omega / n$ :

$$
\mathcal{H}_{3}=-\frac{\mu}{2 a}\left(\frac{\omega}{n}\right)^{3} \gamma^{3} \frac{a^{4}}{r^{4}} s\left[\frac{9}{2}\left(4-5 s^{2}\right) \sin \theta+\frac{15}{2} s^{2} \sin 3 \theta\right]
$$

where

$$
\gamma=\frac{J_{3}^{1 / 3}}{(\omega / n)}(\alpha / a)=\beta \frac{J_{3}^{1 / 3}}{J_{2}^{1 / 2}}
$$

We limit our theory to those regions in phase space in which both $\beta$ and $\gamma$ are of the first order.

We use Deprit's [5] perturbation method to stepwise construct our analytical theory. Basically, Deprit proposes to find a Lie transform $\mathcal{T}: \boldsymbol{y} \rightarrow \boldsymbol{y}^{\prime}$ from "new" to "old" variables such that it transforms an initial Hamiltonian

$$
\mathcal{H}=\sum_{i \geq 0}\left(\epsilon^{i} / i !\right) \mathcal{H}_{i}(\boldsymbol{y})
$$

expanded as a power series of a small parameter $\epsilon$ into a new one

$$
\mathcal{H}^{\prime}=\sum_{i \geq 0}\left(\epsilon^{i} / i !\right) H_{0, i}\left(\boldsymbol{y}^{\prime}\right)
$$

in which, usually, one variable is eliminated after truncation up to a certain order. The transformation is constructed term by term using the recurrence

$$
H_{i, j}=H_{i+1, j-1}+\sum_{0 \leq k \leq i}\left(\begin{array}{c}
i \\
k
\end{array}\right)\left\{H_{k, j-1} ; W_{i+1-k}\right\}
$$

Terms $H_{i, 0}$ are obtained by simply replacing old with new variables in the corresponding terms $\mathcal{H}_{i}$; the structure $\{;\}$ stands for the Poisson bracket:

$$
\{\mathcal{F} ; \mathcal{G}\}=\nabla_{x} \mathcal{F} \cdot \nabla_{X} \mathcal{G}-\nabla_{X} \mathcal{F} \cdot \nabla_{x} \mathcal{G}
$$

where $\mathcal{F}$ and $\mathcal{G}$ are generic functions of the variables $\boldsymbol{y}=(\boldsymbol{x}, \boldsymbol{X})$ (coordinates $\boldsymbol{x}$ and conjugate momenta $\boldsymbol{X}$ ), and the coefficients $W_{i}$ define the generating function of the transformation by its series expansion:

$$
W=\sum_{i \geq 0}\left(\epsilon^{i} / i !\right) W_{i+1}
$$

The transformation equations

$$
\boldsymbol{y}=\sum_{i \geq 0}\left(\epsilon^{i} / i !\right) \boldsymbol{Y}_{0, i}\left(\boldsymbol{y}^{\prime}\right)
$$

are likewise constructed from Eq. (12) by noting that $\boldsymbol{Y}_{0,0}=\boldsymbol{y}^{\prime}$, $\boldsymbol{Y}_{i, 0}=0(i>0)$.

\section{Double-Averaged Problem}

For our perturbation theory, we use Delaunay-type variables $\ell, g$, $h, L, G$, and $H$, where $\ell$ is the mean anomaly and the momenta are functions of the orbital elements:

$$
L=\sqrt{\mu a}, \quad G=L \sqrt{1-e^{2}}, \quad H=G \cos I
$$

The average over the mean anomaly is performed up to the third order by a Lie transform:

$$
\mathcal{T}_{\ell}:(\ell, g, h, L, G, H) \rightarrow\left(\ell^{\prime}, g^{\prime}, h^{\prime}, L^{\prime}, G^{\prime}, H^{\prime}\right)
$$

We start the algorithm by transforming the Hamiltonian equation (ㅁ) into

$$
\mathcal{H}=\sum_{i \geq 0}\left(\epsilon^{i} / i !\right) H_{i, 0}
$$

where $H_{i, 0}=\mathcal{H}_{i}\left(\ell^{\prime}, g^{\prime}, h^{\prime}, L^{\prime}, G^{\prime}, H^{\prime}\right)$ for $i \leq 3$ and $H_{i, 0}=0$ otherwise. After successive applications of Eq. (12), up to the third order, the averaged Hamiltonian over the mean anomaly is

$$
\mathcal{H}^{\prime}=H_{0,0}+\epsilon H_{0,1}+\left(\epsilon^{2} / 2\right) H_{0,2}+\left(\epsilon^{3} / 6\right) H_{0,3}
$$

where $H_{0,0}=-\mu^{2} /\left(2 L^{\prime 2}\right), H_{0,1}=H_{0,0} \varepsilon(2 \sigma)$, and

$$
\begin{aligned}
& H_{0,2}=H_{0,0} \frac{\varepsilon^{2}}{8}\left\{\left(\frac{4 \beta^{\prime 2}}{\eta^{\prime 3}}+2+3 e^{\prime 2}\right)\left(4-6 s^{\prime 2}\right)\right. \\
& +6\left(\frac{12 \beta^{\prime 2}}{5 \eta^{\prime 3}}+2+3 e^{\prime 2}\right) s^{\prime 2} \cos 2 h^{\prime} \\
& +15 e^{\prime 2}\left[2 s^{\prime 2} \cos 2 g^{\prime}+\left(1-c^{\prime}\right)^{2} \cos \left(2 g^{\prime}-2 h^{\prime}\right)\right. \\
& \left.\left.+\left(1+c^{\prime}\right)^{2} \cos \left(2 g^{\prime}+2 h^{\prime}\right)\right]\right\} \\
& H_{0,3}=H_{0,0} \varepsilon^{3} \frac{9}{2} \eta^{\prime}\left\{\frac{\gamma^{\prime 3}}{\eta^{\prime 6}} e^{\prime} s^{\prime}\left(4-5 s^{\prime 2}\right) \sin g^{\prime}\right. \\
& +e^{\prime 2}\left[\frac{5}{4}-\frac{\beta^{\prime 2}}{5 \eta^{\prime 4}} \frac{1+2 \eta^{\prime}}{\left(1+\eta^{\prime}\right)^{2}}\right]\left[\left(1+c^{\prime}\right)^{2} \cos \left(2 g^{\prime}+2 h^{\prime}\right)\right. \\
& \left.\left.-\left(1-c^{\prime}\right)^{2} \cos \left(2 g^{\prime}-2 h^{\prime}\right)\right]\right\}
\end{aligned}
$$

with $\varepsilon=\omega / n^{\prime}, \sigma=H^{\prime} / L^{\prime}=\sqrt{1-e^{\prime 2}} c^{\prime}$, and $\eta=\sqrt{1-e^{2}}$ is the 
eccentricity function. With the prime notation, a prime in any element or function means that it is expressed in the new variables. Thus, $e^{\prime}=\sqrt{1-\left(G^{\prime} / L^{\prime}\right)^{2}}, c^{\prime}=H^{\prime} / G^{\prime}$, and so on.

A new Lie transform

$$
\mathcal{T}_{h}:\left(\ell^{\prime}, g^{\prime}, h^{\prime}, L^{\prime}, G^{\prime}, H^{\prime}\right) \rightarrow\left(\ell^{\prime \prime}, g^{\prime \prime}, h^{\prime \prime}, L^{\prime \prime}, G^{\prime \prime}, H^{\prime \prime}\right)
$$

is used to eliminate the argument of the node. Note that $n^{\prime}=\mu^{2} / L^{\prime 3}$ is constant in the truncated Hamiltonian equation (14). Therefore, the formal parameter $\epsilon$ is not needed any longer, its role being assumed by the real parameter:

$$
\varepsilon=\omega / n^{\prime}=L^{\prime 3} \omega / \mu^{2}
$$

Then we write the Hamiltonian (14) in the double-prime variables as

$$
\mathcal{K}=\sum_{i \geq 0}\left(\varepsilon^{i} / i !\right) K_{i, 0}
$$

where $K_{i, 0}=H_{0, i}\left(-, g^{\prime \prime}, h^{\prime \prime}, L^{\prime \prime}, G^{\prime \prime}, H^{\prime \prime}\right)$ for $i \leq 3$, and $K_{i, 0}=0$ otherwise. After truncation to the third order, we get

$$
\mathcal{K}^{\prime \prime}=K_{0,0}+\varepsilon K_{0,1}+\left(\varepsilon^{2} / 2\right) K_{0,2}+\left(\varepsilon^{3} / 6\right) K_{0,3}
$$

where $K_{0,0}=-\mu^{2} /\left(2 L^{\prime \prime 2}\right), K_{0,1}=2 \sigma^{\prime \prime} K_{0,0}$, and

$$
\left.\begin{array}{l}
K_{0,2}=K_{0,0} \frac{1}{4}\left[\left(\frac{4 \beta^{\prime \prime 2}}{\eta^{\prime \prime 3}}+2+3 e^{\prime \prime 2}\right)\left(2-3 s^{\prime \prime 2}\right)\right. \\
\left.+15 e^{\prime \prime 2} s^{\prime \prime 2} \cos 2 g^{\prime \prime}\right] \\
K_{0,3}=K_{0,0} \frac{9}{8}\left\{\frac{9 \beta^{\prime \prime 2}}{5 \eta^{\prime \prime 5}} \sigma^{\prime \prime} s^{\prime \prime 2}\left(\frac{6 \beta^{\prime \prime 2}}{5 \eta^{\prime \prime 3}}+2+3 e^{\prime \prime 2}\right)\right. \\
+\frac{3}{4} \sigma^{\prime \prime}\left[50 e^{\prime \prime 2}+\left(2-17 e^{\prime \prime 2}\right) s^{\prime \prime 2}\right] \\
+\frac{9}{4}\left(\frac{6 \beta^{\prime \prime 2}}{\eta^{\prime \prime 5}}+5\right) \sigma^{\prime \prime} e^{\prime \prime 2} s^{\prime \prime 2} \cos 2 g^{\prime \prime}+\frac{4 \gamma^{\prime \prime 3}}{\eta^{\prime \prime}} e^{\prime \prime} s^{\prime \prime}\left(4-5 s^{\prime \prime 2}\right) \sin g^{\prime \prime}
\end{array}\right\}
$$

Details on the averaging and the required generating functions, $W^{\ell}$ for averaging the mean anomaly and $W^{h}$ for the elimination of the node, can be found in Appendices A and B.

The double-averaged Hamiltonian equation (19) represents a 1DOF integrable system in the argument of the periapsis and the modulus of the angular momentum vector. The phase space of the integrable reduced problem is made of closed curves and equilibria, the latter corresponding to trajectories that are known as frozen orbits.

\section{Transformation Equations}

For $\xi \in(\ell, g, h, L, G, H)$, the Lie-Deprit algorithm also provides the explicit transformation equations from averaged to nonaveraged variables as power series

$$
\xi=\sum_{i}\left(\epsilon^{i} / i !\right) \xi_{0, i}
$$

in the small parameter $\epsilon$. The coefficients $\xi_{0, i}$ are computed from the recurrence Eq. (12) by replacing $H_{i, j}$ with $\xi_{i, j}$ and taking into account that $\xi_{0,0}=\xi^{\prime}$ and $\xi_{i, 0}=0(i>0)$. It results in

$$
\xi=\xi^{\prime}+\epsilon\left\{\xi^{\prime} ; W_{1}\right\}+\left(\epsilon^{2} / 2\right)\left[\left\{\left\{\xi^{\prime} ; W_{1}\right\} ; W_{1}\right\}+\left\{\xi^{\prime} ; W_{2}\right\}\right]+\cdots
$$

Thus, for given initial conditions $\xi^{\prime \prime}$ in the double-averaged problem, we undo the second transformation

$$
\xi^{\prime}=\xi^{\prime \prime}+\varepsilon \Delta \xi^{\prime \prime}+\left(\varepsilon^{2} / 2\right) \delta \xi^{\prime \prime}+\mathcal{O}\left(\varepsilon^{3}\right)
$$

by computing the first- and second-order corrections

$$
\Delta \xi^{\prime \prime}=\left\{\xi^{\prime \prime} ; W_{1}^{h}\right\}, \quad \delta \xi^{\prime \prime}=\left\{\Delta \xi^{\prime \prime} ; W_{1}^{h}\right\}+\left\{\xi^{\prime \prime} ; W_{2}^{h}\right\}
$$

from Eqs. (B3) and (B6). We obtain the first-order correction $\left(\Delta L^{\prime \prime}=0\right)$

$$
\begin{aligned}
& \Delta \ell^{\prime \prime}=\frac{15}{32}\left\{\left(4-\frac{6}{5} \eta^{\prime \prime 2}-\frac{36 \beta^{\prime \prime 2}}{25 \eta^{\prime \prime}}\right) s^{\prime \prime 2} \sin 2 h^{\prime \prime}\right. \\
& \left.+\left(2-\eta^{\prime \prime 2}\right)\left[\left(1+c^{\prime \prime}\right)^{2} \sin \left(2 g^{\prime \prime}+2 h^{\prime \prime}\right)-\left(1-c^{\prime \prime}\right)^{2} \sin \left(2 g^{\prime \prime}-2 h^{\prime \prime}\right)\right]\right\}
\end{aligned}
$$

$$
\begin{gathered}
\Delta G^{\prime \prime}=\frac{15}{32} L^{\prime \prime} e^{\prime \prime 2}\left[\left(1-c^{\prime \prime}\right)^{2} \cos \left(2 g^{\prime \prime}-2 h^{\prime \prime}\right)\right. \\
\left.-\left(1+c^{\prime \prime}\right)^{2} \cos \left(2 g^{\prime \prime}+2 h^{\prime \prime}\right)\right]
\end{gathered}
$$

$$
\begin{aligned}
& \Delta g^{\prime \prime}=\frac{15}{32 \eta^{\prime \prime}}\left\{\left[2 c^{\prime 2}-\frac{6}{5} \eta^{\prime \prime 2}+\frac{12 \beta^{\prime \prime 2}}{25 \eta^{\prime \prime 3}}\left(5 c^{\prime \prime 2}-3\right)\right] \sin 2 h^{\prime \prime}\right. \\
& -\left(c^{\prime \prime}-\eta^{\prime \prime 2}\right)\left(1-c^{\prime \prime}\right) \sin \left(2 g^{\prime \prime}-2 h^{\prime \prime}\right) \\
& \left.\quad-\left(c^{\prime \prime}+\eta^{\prime \prime 2}\right)\left(1+c^{\prime \prime}\right) \sin \left(2 g^{\prime \prime}+2 h^{\prime \prime}\right)\right\}
\end{aligned}
$$

$$
\begin{aligned}
& \Delta H^{\prime \prime}=-\frac{15}{32} L^{\prime \prime}\left\{\left(2-\frac{6}{5} \eta^{\prime \prime 2}+\frac{24 \beta^{\prime \prime 2}}{25 \eta^{\prime \prime}}\right) s^{\prime \prime 2} \cos 2 h^{\prime \prime}\right. \\
& \left.+e^{\prime \prime 2}\left[\left(1-c^{\prime \prime}\right)^{2} \cos \left(2 g^{\prime \prime}-2 h^{\prime \prime}\right)+\left(1+c^{\prime \prime}\right)^{2} \cos \left(2 g^{\prime \prime}+2 h^{\prime \prime}\right)\right]\right\}
\end{aligned}
$$

$$
\begin{aligned}
& \Delta h^{\prime \prime}=-\frac{15}{32 \eta^{\prime \prime}}\left[\left(2-\frac{6}{5} \eta^{\prime \prime 2}+\frac{24 \beta^{\prime \prime 2}}{25 \eta^{\prime \prime}}\right) c^{\prime \prime} \sin 2 h^{\prime \prime}\right. \\
& \left.-e^{\prime \prime 2}\left(1-c^{\prime \prime}\right) \sin \left(2 g^{\prime \prime}-2 h^{\prime \prime}\right)-e^{\prime 2}\left(1+c^{\prime \prime}\right) \sin \left(2 g^{\prime \prime}+2 h^{\prime \prime}\right)\right]
\end{aligned}
$$

equations that supplement those published in [9] with the correction to the mean anomaly. The second-order corrections of the second transformation involve many more terms and are in Appendix $\underline{\mathrm{C}}$.

Once we recover the long-period perturbations, we can proceed with the next transformation to get the initial conditions in the original problem. Because $W_{1}^{\ell} \equiv 0$, in accordance with Appendix $\underline{\mathrm{A}}$, the second-order transformation equations are

$$
\xi=\xi^{\prime}+\left(\epsilon^{2} / 2\right) \delta \xi^{\prime}+\mathcal{O}\left(\epsilon^{3}\right)
$$

and second-order corrections to Delaunay elements are Poisson series, such as

$$
\begin{aligned}
\delta H^{\prime} & =\frac{\varepsilon^{2}}{32} L^{\prime}\left\{\sum_{l=1,2,3} \sum_{m=-2,0,2} \sum_{n=-2,2} U_{l, m, n} \cos \left(l u^{\prime}+m g^{\prime}+n h^{\prime}\right)\right. \\
& +\frac{24 \beta^{\prime 2}}{5 \eta^{\prime 3}}\left[12 s^{\prime 2}\left(\ell^{\prime}-f^{\prime}\right) \sin 2 h^{\prime}\right. \\
& \left.\left.+\sum_{l=1,2,3} \sum_{m=0,2} \sum_{n=-2,2} F_{l, m, n} \cos \left(l f^{\prime}+m g^{\prime}+n h^{\prime}\right)\right]\right\}
\end{aligned}
$$

$$
\begin{aligned}
\delta h^{\prime} & =\frac{\varepsilon^{2}}{32 \eta^{\prime}}\left\{\sum_{l=1,2,3} \sum_{m=-2,0,2} \sum_{n=-2,0,2} U_{l, m, n} \sin \left(l u^{\prime}+m g^{\prime}+n h^{\prime}\right)\right. \\
& +\frac{8 \beta^{\prime 2}}{5 \eta^{\prime 3}}\left[12 c^{\prime}\left(\ell^{\prime}-f^{\prime}\right)\left(5-3 \cos 2 h^{\prime}\right)\right. \\
& \left.\left.+\sum_{l=1,2,3} \sum_{m=0,2} \sum_{n=-2,2} F_{l, m, n} \sin \left(l f^{\prime}+m g^{\prime}+n h^{\prime}\right)\right]\right\}
\end{aligned}
$$

for which the coefficients $F_{l, m, n}$ and $U_{l, m, n}$ are given (without primes) in Table 1 . 
Table 1 Coefficients $F_{l, m, n}$ and $U_{l, m, n}$ in Eqs. (22) and (23)

\begin{tabular}{|c|c|c|c|c|c|}
\hline & $m$ & $n$ & $l=1$ & $l=2$ & $l=3$ \\
\hline \multicolumn{6}{|c|}{ Equation (22) } \\
\hline \multirow[t]{2}{*}{$F$} & 0 & \pm 2 & $\pm 6 e s^{2}$ & 0 & 0 \\
\hline & 2 & \pm 2 & $\pm 3 e(1 \pm c)^{2}$ & $\pm 3(1 \pm c)^{2}$ & $\pm e(1 \pm c)^{2}$ \\
\hline \multirow[t]{3}{*}{$U$} & 0 & \pm 2 & $\mp 6 e s^{2}$ & $\pm 18 e^{2} s^{2}$ & $\mp 2 e^{3} s^{2}$ \\
\hline & -2 & \pm 2 & $\mp 15 e(1-\eta)^{2}(1 \mp c)^{2}$ & $\pm 3(3+2 \eta)(1-\eta)^{2}(1 \mp c)^{2}$ & $\mp e(1-\eta)^{2}(1 \mp c)^{2}$ \\
\hline & 2 & \pm 2 & $\mp 15 e(1+\eta)^{2}(1 \pm c)^{2}$ & $\pm 3(3-2 \eta)(1+\eta)^{2}(1 \pm c)^{2}$ & $\mp e(1+\eta)^{2}(1 \pm c)^{2}$ \\
\hline \multicolumn{6}{|c|}{ Equation (23) } \\
\hline \multirow[t]{4}{*}{$F$} & 0 & 0 & $-60 c e$ & 0 & 0 \\
\hline & 0 & \pm 2 & $18 c e$ & 0 & 0 \\
\hline & 2 & 0 & $30 c e$ & $30 c$ & $10 c e$ \\
\hline & 2 & \pm 2 & $\mp 9(1 \pm c) e$ & $\mp 9(1 \pm c)$ & $\mp 3(1 \pm c) e$ \\
\hline \multirow[t]{5}{*}{$U$} & 0 & 0 & $12 c e\left(5+3 \eta^{2}\right)$ & $-36 c e^{2}$ & $4 c e^{3}$ \\
\hline & 0 & \pm 2 & $-6 c e\left(5+3 \eta^{2}\right)$ & $18 c e^{2}$ & $-2 c e^{3}$ \\
\hline & \pm 2 & 0 & $-30 c e(1 \pm \eta)^{2}$ & $6 c(3 \mp 2 \eta)(1 \pm \eta)^{2}$ & $-2 c e(1 \pm \eta)^{2}$ \\
\hline & \pm 2 & -2 & $\mp 15(1 \mp c) e(1 \pm \eta)^{2}$ & $\pm 3(1 \mp c)(3 \mp 2 \eta)(1 \pm \eta)^{2}$ & $\mp(1 \mp c) e(1 \pm \eta)^{2}$ \\
\hline & \pm 2 & 2 & $\pm 15(1 \pm c) e(1 \pm \eta)^{2}$ & $\mp 3(1 \pm c)(3 \mp 2 \eta)(1 \pm \eta)^{2}$ & $\pm(1 \pm c) e(1 \pm \eta)^{2}$ \\
\hline
\end{tabular}

The transformation equation for $H$ and $h$, Eqs. (22) and (23), are the shortest Poisson series involved in recovering the short-period effects. Other transformation equations have hundreds of terms and these are omitted, for brevity.

The full transformation equations are, of course, required for computing ephemerides from the analytic theory. However, relevant applications to mission design only require choosing initial conditions from the double-averaged problem and computing the corresponding conditions in the original nonaveraged problem. In such cases, for given values of the integrals $L^{\prime \prime}$ and $H^{\prime \prime}$, a point ( $g$ " and $G^{\prime \prime}$ ) of the double-averaged problem maps onto a torus of the nonaveraged problem. This means that we are free to choose any value of $h^{\prime \prime}$ and $\ell^{\prime \prime}$ for computing the initial conditions in the original problem. We will show that a careful selection of $h^{\prime \prime}$ and $\ell^{\prime \prime}$ dramatically simplifies the transformation equations.

First of all, the mean anomaly is not an argument of the second Lie transformation equations (17-21) and (C1-C6), because it is cyclic after the Delaunay transformation. Therefore, one can trivially set $\ell_{0}^{\prime}=0$ (and $\left.f_{0}^{\prime}=u_{0}^{\prime}=0\right)$. In addition, for given initial conditions $g_{0}^{\prime \prime}$, $G_{0}^{\prime \prime}, H_{0}^{\prime \prime}$, and $L_{0}^{\prime \prime}$, the root-finding problem $h^{\prime} \equiv h^{\prime \prime}+\varepsilon \Delta h^{\prime \prime}\left(h^{\prime \prime}\right)-$ $\left(\varepsilon^{2} / 2\right) \delta h^{\prime \prime}\left(h^{\prime \prime}\right)=0$ will provide a value $h^{\prime \prime}=h_{0}^{\prime \prime}$ such that $h_{0}^{\prime}=0$. The "reduced" $\left(\ell_{0}^{\prime}=h_{0}^{\prime}=0\right)$ transformation equations are

$$
\begin{aligned}
\ell= & -\frac{1}{2} \varepsilon^{2}\left\{\frac{1}{e^{\prime}}\left[\frac{2 \beta^{\prime 2}}{5 \eta^{\prime 2}}\left(4-c^{\prime 2}\right)-4\right]+6-20 e^{\prime}+3 e^{\prime 2}\right\} \eta^{\prime} \sin 2 g^{\prime} \\
g= & g^{\prime}+\frac{1}{2} \varepsilon^{2}\left\{\frac{1}{e^{\prime}}\left[\frac{2 \beta^{\prime 2}}{5 \eta^{\prime 4}}\left(4-c^{\prime 2}\right)-4\right]+\frac{3}{4}+8 e^{\prime}-\frac{9 e^{\prime 2}}{4}\right. \\
& \left.+\frac{\beta^{\prime 2}}{10 \eta^{\prime 4}}\left[3\left(12-5 c^{\prime 2}\right)+16\left(2-c^{\prime 2}\right) e^{\prime}\right]\right\} \sin 2 g^{\prime} \\
L= & L^{\prime}+\frac{1}{2} L^{\prime} \varepsilon^{2}\left\{\frac{\beta^{\prime 2}}{5 \eta^{\prime 6}}\left(2+3 c^{\prime 2}\right)\left(2+\eta^{\prime}\right)\left(1+e^{\prime}\right)\left(1+e^{\prime}-\eta^{\prime}\right)\right. \\
& -\frac{e^{\prime}}{4}\left(4+e^{\prime}\right)+\frac{3}{4}\left[\frac{4 \beta^{\prime 2}}{5 \eta^{\prime 6}}\left(4-c^{\prime 2}\right)\left(1+e^{\prime}\right)^{3}\right) c^{\prime} \sin 2 g^{\prime} \\
& \left.\left.+2-\left(4+3 e^{\prime}\right) e^{\prime}\right] \cos 2 g^{\prime}\right\}
\end{aligned}
$$$$
G=G^{\prime}+\frac{1}{2} G^{\prime} \varepsilon^{2}\left[\frac{\beta^{\prime 2}}{5 \eta^{\prime 4}}\left(4-c^{\prime 2}\right)\left(3+4 e^{\prime}\right)-8 e^{\prime}\right.
$$$$
\left.+\frac{3}{2}\left(1+e^{\prime 2}\right)\right] \cos 2 g^{\prime}
$$

$$
H=H^{\prime}+\frac{1}{2} H^{\prime} \varepsilon^{2}\left[\frac{\beta^{\prime 2}}{5 \eta^{\prime 4}} 3\left(3+4 e^{\prime}\right)-8 e^{\prime}+\frac{3}{2}\left(1+e^{\prime 2}\right)\right] \cos 2 g^{\prime}
$$

Note that Eqs. (24) and (25) contain negative powers of $e^{\prime}$. For $e^{\prime} \sim \varepsilon$, this does not cause trouble, simply meaning that corrections to $g^{\prime}$ and $\ell^{\prime}$ are affected by first-order terms.

Divisions by the eccentricity for smaller values of $e^{\prime}$ should be avoided, and it can be done in different sets of uniformly regular variables [12-14]. Following [12], we use the set $(F, C, S, L, H$, and $h$ ), where $\bar{F}=\bar{\ell}+g$ is the mean distance to the ascending node, and $C=e \cos g$ and $S=e \sin g$ are semi-equinoctial elements.

As for the Hamiltonian, the Lie transform applies to any other function of the coordinates and momenta. Therefore, Deprit's recurrence equation (12) is at hand again to build the transformation equations for $F$ (respectively, $C$ and $S$ ) by simply replacing $H_{i, j}$ with $F_{i, j}$ (respectively, $C_{i, j}$ and $S_{i, j}$ ), where $F_{0,0}=F^{\prime}$ (respectively, $C_{0,0}=C^{\prime}$ and $S_{0,0}=S^{\prime}$ ) and $F_{i, 0}=0$ for $i>0$ (respectively, $C_{i, 0}=$ 0 and $\left.S_{i, 0}=0\right)$. In the new set of elements, the transformation equations (26), (27), and (29) are complemented with

$$
\begin{aligned}
F= & F^{\prime}+\frac{\varepsilon^{2}}{2}\left\{\frac{3}{4}-6 \eta^{\prime}+4\left(2+5 \eta^{\prime}-\frac{1}{1+\eta^{\prime}}\right) e^{\prime}\right. \\
& -\left(\frac{9}{4}+3 \eta^{\prime}\right) e^{\prime 2}+\frac{\beta^{\prime 2}}{10 \eta^{\prime 4}}\left[36-15 c^{\prime 2}-32 e^{\prime}+4\left(4-c^{\prime 2}\right)\right. \\
& \left.\left.\times\left(4+\eta^{\prime}+\frac{1}{1+\eta^{\prime}}\right) e^{\prime}\right]\right\} \sin 2 g^{\prime} \\
C & =C^{\prime}+\frac{\varepsilon^{2}}{2} \frac{1}{4}\left\{\left[\frac{2 \beta^{\prime 2}}{5 \eta^{\prime 4}}\left(24+15 c^{\prime 2}+A_{1} e^{\prime}+\left(16+7 c^{\prime 2}\right) e^{\prime 2}\right)\right.\right. \\
& \left.\left.+14-10 e^{\prime}-22 e^{\prime 2}+13 e^{\prime 3}\right] \cos g^{\prime}+A_{2} \cos 3 g^{\prime}\right\} \\
S= & S^{\prime}+\frac{\varepsilon^{2}}{2} \frac{1}{4}\left\{\left[\frac{2 \beta^{\prime 2}}{5 \eta^{\prime 4}}\left(21 c^{\prime 2}-\left(60-9 c^{\prime 2}-A_{1}\right) e^{\prime}-\left(8-5 c^{\prime 2}\right) e^{\prime 2}\right)\right.\right. \\
& \left.\left.-22+8 e^{\prime}+30 e^{\prime 2}-11 e^{\prime 3}\right] \sin g^{\prime}+A_{2} \sin 3 g^{\prime}\right\}
\end{aligned}
$$

where

$$
A_{1}=42+(27 / 2) c^{\prime 2}+\left(4+6 c^{\prime 2}\right)\left[\eta^{\prime}+1 /\left(1+\eta^{\prime}\right)\right]
$$

$$
\begin{aligned}
A_{2} & =2-6 e^{\prime}+6 e^{\prime 2}+3 e^{\prime 3}+\frac{2 \beta^{\prime 2}}{5 \eta^{\prime 4}}\left[7\left(4-c^{\prime 2}\right)\right. \\
& \left.+\left(66-\frac{39}{2} c^{\prime 2}\right) e^{\prime}+\left(44-15 c^{\prime 2}\right) e^{\prime 2}\right]
\end{aligned}
$$




\section{Application: Computation of the Science Orbit}

The high-inclination, low-altitude orbits required by science missions around planetary satellites are known to be unstable. To maximize the orbit lifetime with minimum control, tours on the stable-unstable manifold of selected frozen orbits in an averaged model have been proposed [7]. The stable-unstable manifold tours can also be designed in the nonaveraged problem [15], in which simple repeat-ground-track orbits were demonstrated to be a feasible option, enjoying long lifetimes even in ephemeris models [16].

To move in average on the stable manifold of an unstable frozen orbit one could choose initial conditions directly from the averaged problem. However, the long and short-period effects that are missed in the averaging will prevent, in general, the desired result. A linearization of the problem allows to compute first-order corrections to recover the averaged effects [7]. On the contrary, the linearization is unnecessary when performing the averaging by Lie transforms. The explicit transformation equations from the averaged to the nonaveraged problem are available as power series in the small parameter. The transformation equations of the elimination of the node allow to recover the long-period perturbations, and those of the averaging of the mean anomaly incorporate the short-period effects.

The computation of stable-unstable manifold tours requires only to choose initial conditions from the double-averaged problem and compute the corresponding ones in the original, nonaveraged problem. Then, in addition to Eqs. (17-21) and (C1-C6), the reduced version Eqs. (24-29) of the first Lie transformation are enough for our purposes. Furthermore, for the low eccentricity and high inclination required by mapping orbits one can neglect higher-order terms, highly simplifying the transformation equations. Thus, up to the second order of this paper, for small enough $h^{\prime \prime}$ and $\ell^{\prime \prime}$ after trivial simplifications, one finds

$$
\begin{gathered}
L=L^{\prime \prime}\left[1+\varepsilon^{\prime \prime 2}(3 / 20)\left(5+8 \beta^{\prime \prime 2}\right) \cos 2 g^{\prime \prime}\right] \\
G=L^{\prime \prime}\left[\eta^{\prime \prime}+\varepsilon^{\prime \prime 2}(3 / 20)\left(5+8 \beta^{\prime \prime 2}\right) \cos 2 g^{\prime \prime}\right] \\
H=L^{\prime \prime}\left[\sigma^{\prime \prime}-\varepsilon^{\prime \prime}(3 / 40)\left(5+6 \beta^{\prime \prime 2}\right)\left(1-\sigma^{\prime \prime 2}\right)\right] \\
\ell=\varepsilon^{\prime \prime}\left[\frac{\varepsilon^{\prime \prime}}{e^{\prime \prime}}\left(2-\frac{4-\sigma^{\prime \prime 2}}{5} \beta^{\prime \prime 2}\right)-3 \varepsilon^{\prime \prime}\right] \sin 2 g^{\prime \prime} \\
g=g^{\prime \prime}-\varepsilon^{\prime \prime}\left[\frac{\varepsilon^{\prime \prime}}{e^{\prime \prime}}\left(2-\frac{4-\sigma^{\prime \prime 2}}{5} \beta^{\prime \prime 2}\right)\right. \\
\left.+\frac{15 \sigma^{\prime \prime}}{8}-\varepsilon^{\prime \prime} \frac{153}{640}\left(5+18 \beta^{\prime \prime 2}\right)\right] \sin 2 g^{\prime \prime} \\
h=0
\end{gathered}
$$

As before, the eccentricity appears in denominators of the transformation equations of the mean anomaly and argument of the periapsis, and the set $(F, C, S, L, H, h)$ of nonsingular elements is recommended for very low-eccentricity orbits, where

$$
\begin{gathered}
F=F^{\prime \prime}-\varepsilon^{\prime \prime 2}(3 / 40)\left(35-24 \beta^{\prime \prime 2}\right) \sin 2 g^{\prime \prime} \\
C=C^{\prime \prime}+\varepsilon^{\prime \prime 2}(1 / 20)\left[\left(35+24 \beta^{\prime \prime 2}\right) \cos g^{\prime \prime}+\left(5+28 \beta^{\prime \prime 2}\right) \cos 3 g^{\prime \prime}\right]
\end{gathered}
$$

$$
S=S^{\prime \prime}-\varepsilon^{\prime \prime 2}(1 / 20)\left[55 \sin g^{\prime \prime}-\left(5+28 \beta^{\prime \prime 2}\right) \sin 3 g^{\prime \prime}\right]
$$

complement the transformation equations (35), (37), and (40).

The long-period corrections to the elements $F^{\prime \prime}, C^{\prime \prime}$, and $\overline{S^{\prime \prime}}$ are of order higher than two for science orbits. Therefore, they are not needed in the computation of Eqs. (41-43), and we do not find it necessary to provide corresponding expressions. Those who prefer to use the full expressions Eqs. (30-32), may recover the long-period terms by computing $F^{\prime}=\ell^{\prime}+g^{\prime}, C^{\prime}=e^{\prime} \cos g^{\prime}$ and $S^{\prime}=e^{\prime} \sin g^{\prime}$ from Eqs. (17-21) and ( $\underline{\mathrm{C} 1}-\mathrm{C} 6)$, which are free from negative powers of the eccentricity.

Equations (35-40) show that for the low-eccentricity and highinclination orbits required by the science mission, the most sensitive orbital elements are the argument of the periapsis and inclination-if one reasonably assumes that a small correction to the mean anomaly does not notably affect the long-term dynamics. Then, a first-order approach is to choose $a^{\prime \prime}, e^{\prime \prime}, I^{\prime \prime}, g^{\prime \prime}$, from the double-averaged problem, set $a=a^{\prime \prime}, e=e^{\prime \prime}, h=\ell=0$, and compute

$$
\begin{gathered}
I=I^{\prime \prime}+\varepsilon \frac{3}{40}\left(5+6 \beta^{2}\right) \sin I^{\prime \prime} \\
g=g^{\prime \prime}-\varepsilon\left[\frac{\varepsilon}{e}\left(2-\frac{4}{5} \beta^{2}\right)+\frac{15}{8} \cos I^{\prime \prime}\right] \sin 2 g^{\prime \prime}
\end{gathered}
$$

Equations (44) are derived from Eqs. (36) and (37) and Eq. (39), in which second-order terms $\varepsilon^{\prime \prime 2}, e^{2}$, and $\bar{\varepsilon}^{\prime \prime} c^{\prime \prime 2}$ were neglected.

As illustration of the procedure, we consider a science orbit around the Galilean moon Europa, where $\mu=3202.7 \mathrm{~km}^{3} / \mathrm{s}^{2}$, $\omega=2.05 \times 10^{-5} \mathrm{rad} / \mathrm{s}, \quad \alpha=1565 \mathrm{~km}, J_{2}=4.355 \times 10^{-4}$ from [17], and $C_{2,2}=(3 / 10) J_{2}$ from synchronous-moon theory.

\section{Central Body with Equatorial Symmetry}

In this example, we do not consider a possible latitudinal asymmetry of Europa and, therefore, frozen orbits exist with zero eccentricity [6]. For a reference circular frozen orbit $120 \mathrm{~km}$ above the surface of Europa and $75 \mathrm{deg}$ of inclination, one gets

$$
L^{\prime \prime}=2323.05 \mathrm{~km}^{2} / \mathrm{s}, \quad H^{\prime \prime}=601.249 \mathrm{~km}^{2} / \mathrm{s}
$$

which produce

$$
\beta^{\prime \prime}=0.773594, \quad \varepsilon^{\prime \prime}=0.0250551, \quad \sigma^{\prime \prime}=0.258819
$$

The corresponding flow in the double-averaged phase space is illustrated in Fig. 1, in which the dotted contour corresponds to the Hamiltonian value $\mathcal{K}$ " of the reference, frozen orbit, and its stable and unstable manifolds obtained after introducing the values of Eqs. (45) and (46) in Eq. (16).

In this flow, orbits with $e>0.071$ impact Europa. We fix an initial eccentricity $e^{\prime \prime}=0.01$ and solve Eq. (16) for the argument of the periapsis. The values $g^{\prime \prime}=143.263$ and $323.263 \mathrm{deg}$ correspond to the two stable manifold branches, whereas $g^{\prime \prime}=36.7369$ and $216.737 \mathrm{deg}$ correspond to the unstable manifolds. We choose $g^{\prime \prime}=323.263 \mathrm{deg}$ and are free to fix any values of $h^{\prime \prime}$ and $\ell^{\prime \prime}$. Setting either $h^{\prime \prime}=\ell^{\prime \prime}=0$ or $h^{\prime \prime}=0.000135 \mathrm{deg}, \ell^{\prime \prime}=0.6 \mathrm{deg}$ makes no appreciable differences when running these initial conditions in the

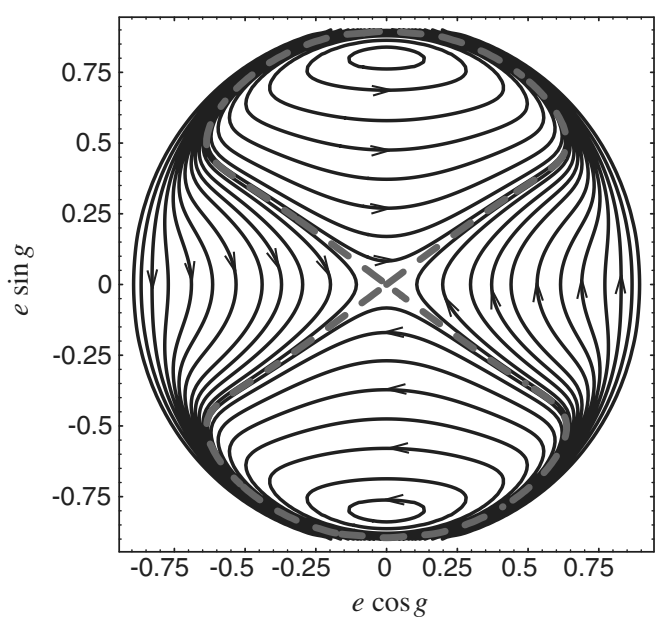

Fig. 1 Flow in the double-averaged problem $\left(J_{3}=0\right)$. 
Table 2 Initial orbital elements of the science orbit

\begin{tabular}{lllcccc}
\hline \hline Corrections & $a, \mathrm{~km}$ & \multicolumn{1}{c}{$e$} & \multicolumn{1}{c}{$I, \mathrm{deg}$} & $g, \mathrm{deg}$ & \multicolumn{1}{c}{$h, \mathrm{deg}$} & \multicolumn{1}{c}{, $\mathrm{deg}$} \\
\hline None & 1685. & 0.01 & 74.9992 & 323.263 & 0.00013484 & 0.600404 \\
Equation (44) & 1685. & 0.01 & 75.9568 & 329.177 & 0. & 0. \\
Equations (ㅍ-40) & 1685.88 & 0.009999 & 75.8946 & 329.074 & 0. & -5.16974 \\
\hline \hline
\end{tabular}

nonaveraged problem. In both cases the orbit follows roughly the stable-unstable manifold path and impacts the surface of Europa after about eight weeks, remaining with $e \leq 0.01$ around four weeks. However, the latter result in $h^{\prime}=\ell^{\prime}=0$, allowing us to use the simplified first- or second-order Eqs. (35-40) and (44) for the transformation from the double-averaged to the nonaveraged problem.

Thus, the first-order corrections of Eq. (44) provide initial conditions of an orbit of the original problem that, in average, evolves much closer to the manifolds of the double-averaged problem, improving lifetime up to more than 16 weeks, approximately 12 of which the eccentricity is below the initial value. An impressive improvement is found when using Eqs. (3540) instead of Eq. (44), remaining four months and a half with $\bar{e} \leq 0.01$ and with more than five months lifetime.

Table 2 summarizes the orbital elements after the different corrections, and Fig. 2 shows the radius evolution in the three different cases. The final evolution of the orbit in the reduced phase space is presented in Fig. 3 .

\section{Central Body with Latitudinal Asymmetry}

We choose the same values of the parameters $L^{\prime \prime}$ and $H^{\prime \prime}$ given in Eq. (45) and, consequently, the values of $\beta^{\prime \prime}, \varepsilon^{\prime \prime}$, and $\sigma^{\prime \prime}$ given in Eq. ( $\overline{46}$ ). The latitudinal asymmetry makes evident by a nonzero $J_{3}$ value. Lacking of information about Europa's $J_{3}$ and with the only

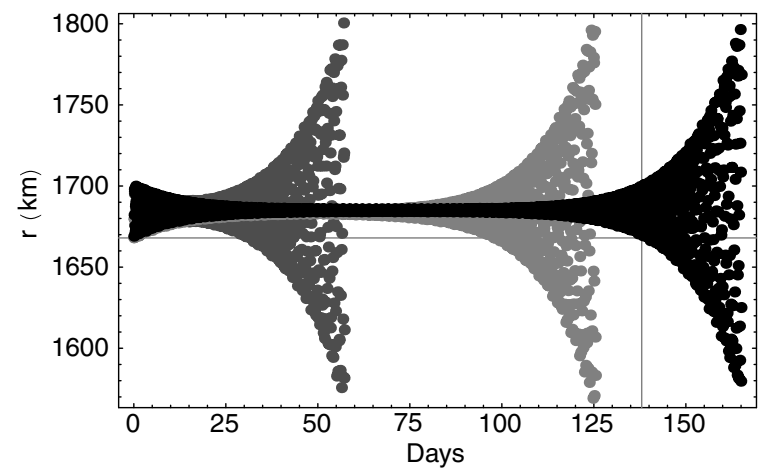

Fig. 2 Radius evolution after different corrections $\left(J_{3}=0\right)$.

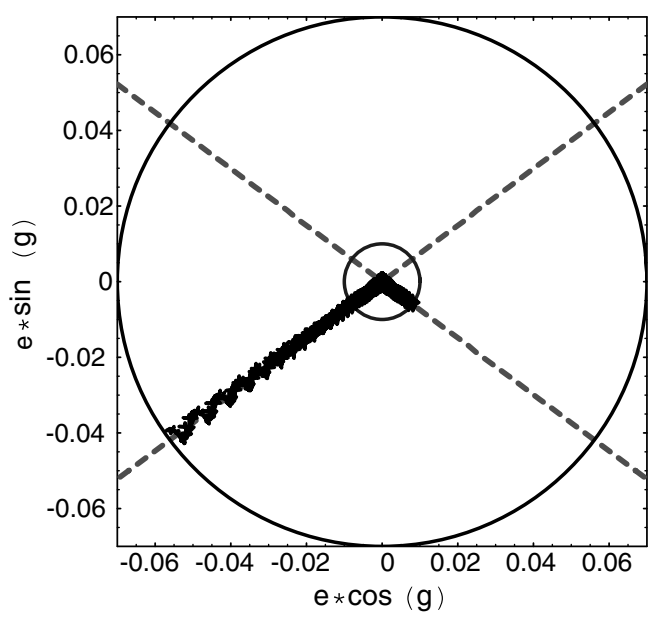

Fig. 3 Final behavior in the reduced phase space $\left(J_{3}=0\right)$. purpose of providing an example we choose $J_{3}=2 \times 10^{-5}$, which results in $\gamma \approx 1$ clearly satisfying the requirements of our theory of being a third-order effect.

A detail of the reduced phase space of this example is illustrated in Fig. 4 in which we clearly appreciate an unstable frozen orbit with small but nonzero eccentricity and argument of the periapsis at $-\pi / 2$. The dotted line corresponds to the stable and unstable manifolds of the frozen orbit.

To show the utility of the simplified formulas in nonsingular elements, we apply them to transform the initial conditions of the frozen orbit $e^{\prime \prime}=0.00270285$ and $g^{\prime \prime}=-\pi / 2$, instead of choosing an orbit of the stable manifold with higher eccentricity. Without undoing the transformations, the propagation of corresponding initial conditions in the original problem results in an impact orbit on the surface of Europa after about 46 days. However, after computing second-order correction using the nonsingular simplified transformation equations (35), (37), and (40-43) for the double-averaged elements $F^{\prime \prime}=g^{\prime \prime}, C^{\prime \prime}=0, \bar{S}^{\prime \prime}=-e^{\prime \prime}$, and $h^{\prime \prime}=0$ and the values of $L^{\prime \prime}$ and $H^{\prime \prime}$ given in Eq. (45), the orbit remains with almost constant eccentricity for about four months and impacts Europa one month later.

Table 3 presents initial conditions taken from the double-averaged problem and those after recovering the long and short-period effects. Figure 5 shows the radius evolution in both cases.

We must say that our theory does not provide any control over which branch of the unstable manifold will escape the orbit. Strategies based on the introduction of small corrections to the argument of the periapsis may be developed to provide such a control [7].

Tests on a variety of examples (including both cases $J_{3}=0$ and $J_{3} \neq 0$ ) show that lifetimes can vary, ranging from around four months to the impressive performances of the examples provided. But improvement is always found when using either the full or the simplified transformation equations for computing initial conditions of the nonaveraged problem. Note that we do not claim to find the longest lifetimes orbits. Because of the importance of higher-order effects in the unstable dynamics, longer lifetimes may be obtained either analytically with a higher-order theory, or numerically with a final tuning of the argument of the periapsis [7]. Alternatively, unstable repeat ground track orbits of the nonaveraged problem were demonstrated to be a feasible option, enjoying long lifetimes even in ephemeris models [16].

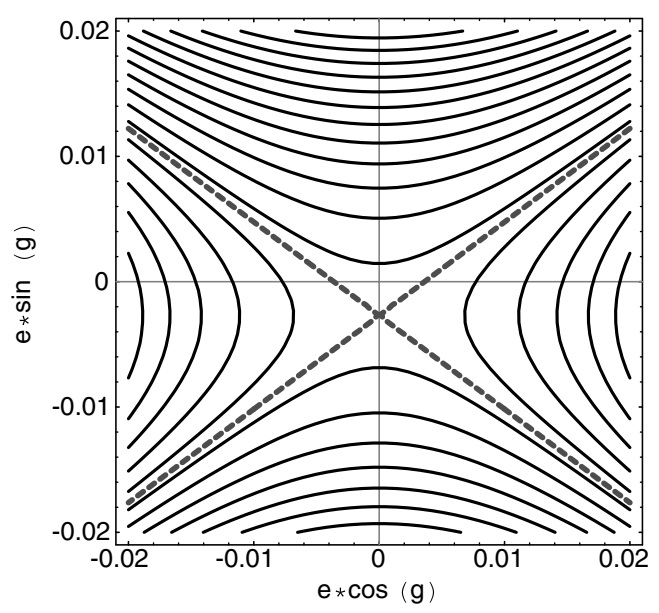

Fig. 4 Flow in the reduced phase space $\left(J_{3} \neq 0\right)$. 
Table 3 Initial orbital elements of the frozen orbit

\begin{tabular}{lcccccc}
\hline \hline Corrections & $a, \mathrm{~km}$ & $e$ & $I, \mathrm{deg}$ & $g, \mathrm{deg}$ & $h, \mathrm{deg}$ & $\ell, \mathrm{deg}$ \\
\hline None & 1685. & 0.0027 & 74.9999 & $-\pi / 2$ & 0 & 0 \\
Equations (25), (37), and (40-43) & 1681.90 & 0.0003 & 75.8783 & $-\pi / 2$ & 0 & 0 \\
\hline \hline
\end{tabular}

\section{Conclusions}

Future missions to planetary satellites shall deal with unstable dynamics. Accurate values of the harmonic coefficients of prospective planetary satellite targets will not be definitely known until the orbiter performs a variety of measures as part of the science mission itself. Therefore, literal expressions coming from analytical theories may help in the design of science missions. Notably, certain maneuvers are easily designed in the double-averaged problem, and the evaluation of the simplified transformation equations of the averaging given in this paper provides accurate initial conditions in the nonaveraged problem in a straightforward way.

The theory presented here is complete up to the second order in the small parameter. However, it must be noted that second-order terms produce first-order corrections to the argument of the periapsis of low-eccentricity orbits, which is a highly sensitive element when dealing with the stable-unstable manifold dynamics. Analogously, second-order corrections to the argument of the periapsis of loweccentricity orbits are expected from third-order terms, and it may worthwhile to develop a higher-order theory than that presented here.

\section{Appendix A: Averaging the Mean Anomaly}

The Hamiltonian equation (5) is written as

$$
\mathcal{H}=\sum_{i \geq 0}\left(\epsilon^{i} / i !\right) H_{i, 0}
$$

where the terms $H_{i, 0}=\mathcal{H}_{i}\left(\ell^{\prime}, g^{\prime}, h^{\prime}, L^{\prime}, G^{\prime}, H^{\prime}\right)$ for $i \leq 3$ and $H_{i, 0}=$ 0 otherwise. For the first order, Eq. (12) gives

$$
H_{0,1}=H_{1,0}+\left\{H_{0,0} ; W_{1}^{\ell}\right\}=-\omega H^{\prime}-\frac{\mu^{2}}{L^{\prime 3}} \frac{\partial W_{1}^{\ell}}{\partial \ell^{\prime}}
$$

where we assigned a superscript to the coefficients of the generating function to manifest that it corresponds to the transformation that averages the mean anomaly. Because $H_{1,0}$ does not depend on $\ell$, we trivially choose $W_{1}^{\ell} \equiv 0$ and set $H_{0,1}=H_{1,0}$, where $H_{1,0}$ is expressed in the prime variables $H_{0,1}=H_{0,0}\left(\omega / n^{\prime}\right)\left(2 \sqrt{1-e^{\prime 2}} c^{\prime}\right)$. Therefore, up to the first order, the transformation is the identity.

The second order of Deprit's recurrence, Eq. (12), gives $H_{0,2}=$ $H_{1,1}+\left\{H_{0,1} ; W_{1}^{\ell}\right\}$ and $H_{1,1}=H_{2,0}+\left\{H_{1,0} ; \overline{W_{1}^{\ell}}\right\}+\left\{H_{0,0} ; W_{2}^{\ell}\right\}$. Because $W_{1}^{\ell} \equiv 0$ in our theory,

$$
H_{0,2}=H_{1,1}=H_{2,0}+\left\{H_{0,0} ; W_{2}^{\ell}\right\}=H_{2,0}-\frac{\mu^{2}}{L^{\prime 3}} \frac{\partial W_{2}^{\ell}}{\partial \ell^{\prime}}
$$

We choose $H_{0,2}=\left\langle H_{2,0}\right\rangle_{\ell^{\prime}}$ as the average of $H_{2,0}$ over the mean anomaly $\ell^{\prime}$, which is an implicit variable in $H_{2,0}$. Part of the

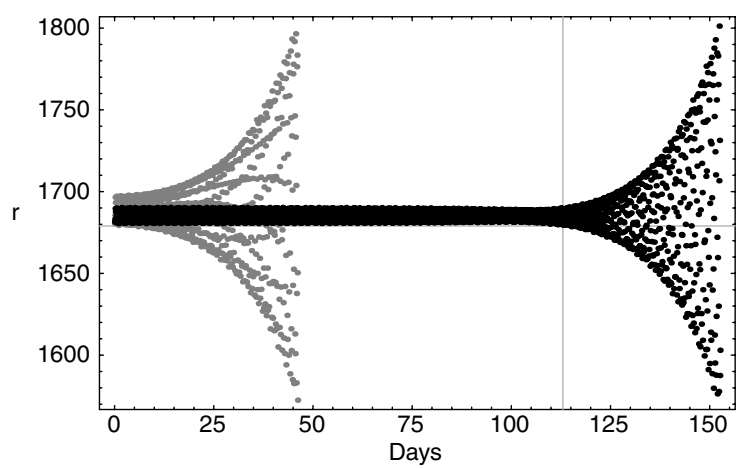

Fig. 5 Radius evolution after second-order nonsingular simplified corrections $\left(J_{3} \neq 0\right)$. perturbing function is directly proportional to $r$ (third-body contribution) and part is inversely proportional to it (nonsphericity effect). Therefore, it is convenient to compute the necessary quadratures in two steps:

$$
\left\langle H_{2,0}\right\rangle_{\ell^{\prime}}=\int_{0}^{2 \pi} H_{2,0, f} \mathrm{~d} \ell^{\prime}+\int_{0}^{2 \pi} H_{2,0, u} \mathrm{~d} \ell^{\prime}
$$

where

$$
\begin{aligned}
& H_{2,0, f}=H_{0,0}\left(\omega / n^{\prime}\right)^{2} \beta^{\prime 2}\left(a^{\prime} / r^{\prime}\right)^{3}\left\{2-3 s^{\prime 2}+3 s^{\prime 2} \cos 2 \theta^{\prime}\right. \\
& \quad+(9 / 10)\left[2 s^{\prime 2} \cos 2 h^{\prime}+\left(1-c^{\prime}\right)^{2} \cos \left(2 h^{\prime}-2 \theta^{\prime}\right)\right. \\
& \left.\left.\quad+\left(1+c^{\prime}\right)^{2} \cos \left(2 h^{\prime}+2 \theta^{\prime}\right)\right]\right\} \\
& H_{2,0, u}=H_{0,0}\left(\omega / n^{\prime}\right)^{2}(1 / 2)\left(r^{\prime} / a^{\prime}\right)^{2}\left\{2-3 s^{\prime 2}+3 s^{\prime 2} \cos 2 \theta^{\prime}\right. \\
& \quad+(3 / 2)\left[2 s^{\prime 2} \cos 2 h^{\prime}+\left(1-c^{\prime}\right)^{2} \cos \left(2 h^{\prime}-2 \theta^{\prime}\right)\right. \\
& \left.\left.\quad+\left(1+c^{\prime}\right)^{2} \cos \left(2 h^{\prime}+2 \theta^{\prime}\right)\right]\right\}
\end{aligned}
$$

The quadrature

$$
\int_{0}^{2 \pi} H_{2,0, f} \mathrm{~d} \ell^{\prime}
$$

is solved in the true anomaly doing the change $a^{2} \sqrt{1-e^{2}} \mathrm{~d} \ell=r^{2} \mathrm{~d} f$. On the contrary, the change $\mathrm{d} \ell=(1-e \cos u) \mathrm{d} u$ and the ellipse relations $\quad r \sin f=a \sqrt{1-e^{2}} \sin u$ and $\quad r \cos f=a(\cos u-e)$ permit us to integrate the quadrature

$$
\int_{0}^{2 \pi} H_{2,0, u} \mathrm{~d} \ell^{\prime}
$$

in the elliptic anomaly. Thus,

$$
\begin{aligned}
& H_{0,2}=H_{0,0} \frac{1}{8}\left(\frac{\omega}{n^{\prime}}\right)^{2}\left\{\left(\frac{4 \beta^{\prime 2}}{\eta^{\prime 3}}+2+3 e^{\prime 2}\right)\left(4-6 s^{\prime 2}\right)\right. \\
& +6\left(\frac{12 \beta^{\prime 2}}{5 \eta^{\prime 3}}+2+3 e^{\prime 2}\right) s^{\prime 2} \cos 2 h^{\prime}+15 e^{\prime 2}\left[2 s^{\prime 2} \cos 2 g^{\prime}\right. \\
& \left.\left.+\left(1-c^{\prime}\right)^{2} \cos \left(2 g^{\prime}-2 h^{\prime}\right)+\left(1+c^{\prime}\right)^{2} \cos \left(2 g^{\prime}+2 h^{\prime}\right)\right]\right\}
\end{aligned}
$$

where $\eta=\sqrt{1-e^{2}}$ is the eccentricity function.

Once the Hamiltonian term $H_{0,2}$ is chosen, the generator $W_{2}^{\ell}$ can be computed from Eq. (A1) as $W_{2}^{\ell}=\left(1 / \mu^{2}\right) L^{\prime 3} \int\left(H_{2,0}-H_{0,2}\right) \mathrm{d} \ell^{\prime}$, where, again, the quadrature is performed in two steps. We obtain

$$
\begin{aligned}
W_{2}^{\ell} & =\left(\frac{\omega}{n^{\prime}}\right)^{2} \frac{L^{\prime}}{64} \sum_{l=1,2,3} \sum_{m=-2,0,2} \sum_{n=-2,0,2} U_{l, m, n} \sin \left(l u^{\prime}+m g^{\prime}+n h^{\prime}\right) \\
& -\left(\frac{\omega}{n^{\prime}}\right)^{2} \frac{L^{\prime} \beta^{\prime 2}}{10 \eta^{\prime 3}}\left[\left(f^{\prime}-\ell^{\prime}\right)\left(10-15 s^{\prime 2}+9 s^{\prime 2} \cos 2 h^{\prime}\right)\right. \\
& \left.+\frac{1}{4} \sum_{l=1,2,3} \sum_{m=0,2} \sum_{n=-2,0,2} F_{l, m, n} \sin \left(l f^{\prime}+m g^{\prime}+n h^{\prime}\right)\right]
\end{aligned}
$$

for which the coefficients $F_{l, m, n}$ and $U_{l, m, n}$, are given (without primes) in Table A1.

Despite pursuing a second-order theory, it will be soon apparent that the third-order term of the averaged Hamiltonian over the mean anomaly is necessary if we want to reach the second order in the 
Table A1 Coefficients $F_{l, m, n}$ and $U_{l, m, n}$ in Eq. (A5)

\begin{tabular}{cccccc}
\hline \hline & $m$ & $n$ & $l=1$ & $l=2$ & $l=3$ \\
\hline$F$ & 0 & 0 & $20\left(2-3 s^{2}\right) e$ & 0 & 0 \\
& 2 & 0 & $30 e s^{2}$ & $30 s^{2}$ & $10 e s^{2}$ \\
& 0 & \pm 2 & $18 e s^{2}$ & 0 & 0 \\
& 2 & \pm 2 & $9 e(1 \pm c)^{2}$ & $9(1 \pm c)^{2}$ & $3 e(1 \pm c)^{2}$ \\
$U$ & 0 & 0 & $4 e\left(2-3 s^{2}\right)\left(5+3 \eta^{2}\right)$ & $-12 e^{2}\left(2-3 s^{2}\right)$ & $(4 / 3) e^{3}\left(2-3 s^{2}\right)$ \\
& \pm 2 & 0 & $30 e s^{2}(1 \pm \eta)^{2}$ & $-6 s^{2}(1 \pm \eta)^{2}(3 \mp 2 \eta)$ & $2 e s^{2}(1 \pm \eta)^{2}$ \\
& 0 & \pm 2 & $6 e s^{2}\left(5+3 \eta^{2}\right)$ & $-18 e^{2} s^{2}$ & $2 e^{3} s^{2}$ \\
& \pm 2 & -2 & $15(1 \mp c)^{2} e(1 \pm \eta)^{2}$ & $-3(1 \mp c)^{2}(1 \pm \eta)^{2}(3 \mp 2 \eta)$ & $(1 \mp c)^{2} e(1 \pm \eta)^{2}$ \\
& \pm 2 & 2 & $15(1 \pm c)^{2} e(1 \pm \eta)^{2}$ & $-3(1 \pm c)^{2}(1 \pm \eta)^{2}(3 \mp 2 \eta)$ & $(1 \pm c)^{2} e(1 \pm \eta)^{2}$ \\
\hline \hline
\end{tabular}

elimination of the node [12]. After simplifications, the third order of Deprit's recurrence gives

$$
H_{0,3}=H_{3,0}+3\left\{H_{1,0} ; W_{2}^{\ell}\right\}-\frac{\mu^{2}}{L^{\prime 3}} \frac{\partial W_{3}^{\ell}}{\partial \ell^{\prime}}
$$

where we choose $H_{0,3}$ as the average $H_{0,3}=\left\langle H_{3,0}+3\left\{H_{1,0} ; W_{2}^{\ell}\right\}\right\rangle_{\ell^{\prime}}$. Again, we find terms that are better integrated either in the true or the eccentric anomaly. After some computations, we obtain

$$
\begin{aligned}
& H_{0,3}=H_{0,0}\left(\frac{\omega}{n^{\prime}}\right)^{3} \frac{9}{2} \eta^{\prime}\left\{\frac{\gamma^{\prime 3}}{\eta^{\prime 6}} e^{\prime} s^{\prime}\left(4-5 s^{\prime 2}\right) \sin g^{\prime}\right. \\
& +e^{\prime 2}\left[\frac{5}{4}-\frac{\beta^{\prime 2}}{5 \eta^{\prime 4}} \frac{1+2 \eta^{\prime}}{\left(1+\eta^{\prime}\right)^{2}}\right]\left[\left(1+c^{\prime}\right)^{2} \cos \left(2 g^{\prime}+2 h^{\prime}\right)\right. \\
& \left.\left.\quad-\left(1-c^{\prime}\right)^{2} \cos \left(2 g^{\prime}-2 h^{\prime}\right)\right]\right\}
\end{aligned}
$$

After truncation to the third order, the averaged Hamiltonian over the mean anomaly

$$
\mathcal{H}^{\prime}=H_{0,0}+\epsilon H_{0,1}+\left(\epsilon^{2} / 2\right) H_{0,2}+\left(\epsilon^{3} / 6\right) H_{0,3}
$$

is cyclic in $\ell^{\prime}$ and, therefore, the conjugate momentum $L^{\prime}$ is an integral. Then the coupled motion of $g^{\prime}, h^{\prime}, G^{\prime}$, and $H^{\prime}$ decouples from $\ell^{\prime}$; after solving the Hamilton equations of the 2-DOF problem

$$
\begin{array}{rlrl}
\frac{\mathrm{d} g^{\prime}}{\mathrm{d} t}=\frac{\partial \mathcal{H}^{\prime}}{\partial G^{\prime}}, & \frac{\mathrm{d} h^{\prime}}{\mathrm{d} t} & =\frac{\partial \mathcal{H}^{\prime}}{\partial H^{\prime}}, & \frac{\mathrm{d} G^{\prime}}{\mathrm{d} t}=-\frac{\partial \mathcal{H}^{\prime}}{\partial g^{\prime}} \\
\frac{\mathrm{d} H^{\prime}}{\mathrm{d} t} & =-\frac{\partial \mathcal{H}^{\prime}}{\partial h^{\prime}}
\end{array}
$$

the mean anomaly is computed from the quadrature

$$
\ell^{\prime}=\ell_{0}^{\prime}+\int \frac{\partial}{\partial L^{\prime}} \mathcal{H}^{\prime}\left(g^{\prime}(t), h^{\prime}(t), G^{\prime}(t), H^{\prime}(t) ; L^{\prime}\right) \mathrm{d} t
$$

\section{Appendix B: Elimination of the Argument of the Node}

We write the averaged Hamiltonian over the mean anomaly, Eq. (14), in the double-prime variables as

$$
\mathcal{K}=\sum_{i \geq 0}\left(\varepsilon^{i} / i !\right) K_{i, 0}
$$

where $K_{i, 0}=H_{0, i}\left(-, g^{\prime \prime}, h^{\prime \prime}, L^{\prime \prime}, G^{\prime \prime}, H^{\prime \prime}\right)$ for $i \leq 3$, and $K_{i, 0}=0$ otherwise.

To the first order, Deprit's recurrence gives $K_{0,1}=$ $K_{1,0}+\left\{K_{0,0} ; W_{1}^{h}\right\}$. But $W_{1}^{h}$ cannot be computed at this order because the needed generating function $W^{h}$ does not depend on the mean anomaly (although the new Lie transformation indeed does apply to it) and

$$
\left\{K_{0,0} ; W_{i}^{h}\right\}=-\left(\mu^{2} / L^{\prime \prime 3}\right)\left(\partial W_{i}^{h} / \partial \ell^{\prime \prime}\right) \equiv 0
$$

at any order $i$. Then we set $K_{0,1}=K_{1,0}$ and proceed to the second order, where we find

$$
K_{0,2}=K_{2,0}+2 \frac{\mu^{2}}{L^{\prime \prime 3}} \frac{\partial W_{1}^{h}}{\partial h^{\prime \prime}}
$$

We choose $K_{0,2}$ as the average of $K_{2,0}$ over $h^{\prime \prime}$

$$
K_{0,2}=K_{0,0} \frac{1}{4}\left[\left(\frac{4 \beta^{\prime \prime 2}}{\eta^{\prime \prime 3}}+2+3 e^{\prime \prime 2}\right)\left(2-3 s^{\prime \prime 2}\right)+15 e^{\prime \prime 2} s^{\prime \prime 2} \cos 2 g^{\prime \prime}\right]
$$

and compute $W_{1}^{h}$ from Eq. (스):

$$
\begin{aligned}
& W_{1}^{h}=\frac{3 L^{\prime \prime}}{64}\left\{\left(\frac{24 \beta^{\prime \prime 2}}{5 \eta^{\prime \prime 3}}+4+6 e^{\prime \prime 2}\right) s^{\prime \prime 2} \sin 2 h^{\prime \prime}\right. \\
& \left.+5 e^{\prime \prime 2}\left[\left(1+c^{\prime \prime}\right)^{2} \sin \left(2 g^{\prime \prime}+2 h^{\prime \prime}\right)-\left(1-c^{\prime \prime}\right)^{2} \sin \left(2 g^{\prime \prime}-2 h^{\prime \prime}\right)\right]\right\}
\end{aligned}
$$

Analogously,

$$
K_{0,3}=K_{3,0}+\left\{2 K_{2,0}+\left\{K_{1,0} ; W_{1}^{h}\right\}+K_{0,2} ; W_{1}^{h}\right\}+3 \frac{\mu^{2}}{L^{\prime \prime 3}} \frac{\partial W_{2}^{h}}{\partial h^{\prime \prime}}
$$

We choose $K_{0,3}$ as the average over $h^{\prime \prime}$ of the known terms in Eq. (B4):

$$
\begin{aligned}
& K_{0,3}=K_{0,0} \frac{9}{8}\left\{\frac{9 \beta^{\prime \prime 2}}{5 \eta^{\prime \prime}} \sigma^{\prime \prime} s^{\prime \prime 2}\left(\frac{6 \beta^{\prime \prime 2}}{5 \eta^{\prime \prime 3}}+2+3 e^{\prime \prime 2}\right)\right. \\
& +\frac{3}{4} \sigma^{\prime \prime}\left[50 e^{\prime \prime 2}+\left(2-17 e^{\prime \prime 2}\right) s^{\prime \prime 2}\right]+\frac{9}{4}\left(\frac{6 \beta^{\prime \prime 2}}{\eta^{\prime \prime}}+5\right) \sigma^{\prime \prime} e^{\prime \prime 2} s^{\prime 2} \cos 2 g^{\prime \prime} \\
& \left.+\frac{4 \gamma^{\prime \prime 3}}{\eta^{\prime \prime 5}} e^{\prime \prime} s^{\prime \prime}\left(4-5 s^{\prime \prime 2}\right) \sin g^{\prime \prime}\right\}
\end{aligned}
$$

where $\sigma=H / L=c \eta$. Finally, $W_{2}^{h}$ is computed from Eq. (B4) as

$$
\begin{aligned}
W_{2}^{h} & =-\frac{3 L^{\prime \prime} \eta^{\prime \prime}}{128}\left\{6 c ^ { \prime \prime } \left[\frac{16 \beta^{\prime \prime 2}}{5 \eta^{\prime \prime 5}}\left(\frac{3 \beta^{\prime \prime 2}}{2 \eta^{\prime \prime 3}}+2+3 e^{\prime \prime 2}\right)\right.\right. \\
& \left.+2-17 e^{\prime \prime 2}\right] s^{\prime \prime 2} \sin 2 h^{\prime \prime}+\left[\frac { 2 \beta ^ { \prime \prime 2 } } { 5 \eta ^ { \prime \prime 5 } } \left(46-\frac{16+24 \eta^{\prime \prime}}{\left(1+\eta^{\prime \prime}\right)^{2}}\right.\right. \\
& \left.\left.-105 c^{\prime \prime}+75 s^{\prime \prime 2}\right)+5\left(2-9 c^{\prime \prime}\right)\right] e^{\prime \prime 2}\left(1+c^{\prime \prime}\right)^{2} \sin \left(2 g^{\prime \prime}+2 h^{\prime \prime}\right) \\
& +\left[\frac{2 \beta^{\prime \prime 2}}{5 \eta^{\prime \prime 5}}\left(46-\frac{16+24 \eta^{\prime \prime}}{\left(1+\eta^{\prime \prime}\right)^{2}}+105 c^{\prime \prime}+75 s^{\prime \prime 2}\right)\right. \\
& \left.\left.+5\left(2+9 c^{\prime \prime}\right)\right] e^{\prime \prime 2}\left(1-c^{\prime \prime}\right)^{2} \sin \left(2 g^{\prime \prime}-2 h^{\prime \prime}\right)\right\}
\end{aligned}
$$

After truncation to the third order, the double-averaged Hamiltonian equation (16), 


$$
\mathcal{K}^{\prime \prime}=K_{0,0}+\varepsilon K_{0,1}+\left(\varepsilon^{2} / 2\right) K_{0,2}+\left(\varepsilon^{3} / 6\right) K_{0,3}
$$

represents an integrable system in which the 1-DOF motion of the argument of the periapsis and the modulus of the angular momentum vector,

$$
\frac{\mathrm{d} g^{\prime \prime}}{\mathrm{d} t}=\frac{\partial \mathcal{K}^{\prime \prime}}{\partial G^{\prime \prime}}, \quad \frac{\mathrm{d} G^{\prime \prime}}{\mathrm{d} t}=-\frac{\partial \mathcal{K}^{\prime \prime}}{\partial g^{\prime \prime}}
$$

decouples from $h^{\prime \prime}$ and $\ell^{\prime \prime}$, which are integrated from the quadratures

$$
\begin{aligned}
& h^{\prime \prime}=h_{0}^{\prime \prime}+\int \frac{\partial}{\partial H^{\prime \prime}} \mathcal{K}^{\prime \prime}\left[g^{\prime \prime}(t), G^{\prime \prime}(t) ; H^{\prime \prime}, L^{\prime \prime}\right] \mathrm{d} t \\
& \ell^{\prime \prime}=\ell_{0}^{\prime \prime}+\int \frac{\partial}{\partial L^{\prime \prime}} \mathcal{K}^{\prime \prime}\left[g^{\prime \prime}(t), G^{\prime \prime}(t) ; H^{\prime \prime}, L^{\prime \prime}\right] \mathrm{d} t
\end{aligned}
$$

\section{Appendix C: Long-Period Terms and Second-Order Corrections}

Next we detail the second-order corrections of the Lie transformation related to the elimination of the node. The doubleprime notation is omitted in the right side of the equations without risk of confusion.

$$
\delta L^{\prime \prime}=0
$$

$$
\begin{aligned}
& \delta G^{\prime \prime}=\frac{15 L e^{2}}{512 \eta}\left\{30\left[1-c^{4}-2\left(1+3 c^{2}\right) \eta^{2}\right]-6 s^{2}\left[\frac{6 \beta^{2}}{5 \eta^{3}}\left(1+5 c^{2}\right)\right.\right. \\
& \left.-5 s^{2}+6 \eta^{2}\right] \cos 2 g+8\left[(2+9 c) \eta^{2}+\frac{2 \beta^{2}}{25 \eta^{3}}(121+105 c\right. \\
& \left.\left.-75 c^{2}-\frac{8(2+3 \eta)}{(1+\eta)^{2}}\right)\right](1-c)^{2} \cos (2 g-2 h) \\
& +8\left[(2-9 c) \eta^{2}+\frac{2 \beta^{2}}{25 \eta^{3}}\left(121-105 c-75 c^{2}\right.\right. \\
& \left.\left.-\frac{8(2+3 \eta)}{(1+\eta)^{2}}\right)\right](1+c)^{2} \cos (2 g+2 h)+30 s^{4} \cos 4 h \\
& +15 s^{2}\left(1+\frac{6 \beta^{2}}{5 \eta^{3}}\right)(1-c)^{2} \cos (2 g-4 h) \\
& \left.+15 s^{2}\left(1+\frac{6 \beta^{2}}{5 \eta^{3}}\right)(1+c)^{2} \cos (2 g+4 h)\right\}
\end{aligned}
$$

$$
\begin{aligned}
\delta H^{\prime \prime} & =-\frac{3 L \eta}{64}\left\{\frac{36 \beta^{2}}{5 \eta^{5}} c s^{2}\left(\frac{6 \beta^{2}}{5 \eta^{3}}+5-3 \eta^{2}\right)+3 c\left[5\left(7+3 c^{2}\right)\right.\right. \\
& \left.-\left(33+17 c^{2}\right) \eta^{2}\right]+45 c e^{2} s^{2}\left(1+\frac{6 \beta^{2}}{5 \eta^{5}}\right) \cos 2 g \\
& -6 c s^{2}\left[\frac{8 \beta^{2}}{5 \eta^{5}}\left(10-6 \eta^{2}+\frac{3 \beta^{2}}{\eta^{3}}\right)-15+17 \eta^{2}\right] \cos 2 h \\
& +5 e^{2}\left[2+9 c+\frac{2 \beta^{2}}{25 \eta^{5}}\left(121+105 c-75 c^{2}\right.\right. \\
& \left.\left.-\frac{8(2+3 \eta)}{(1+\eta)^{2}}\right)\right](1-c)^{2} \cos (2 g-2 h) \\
- & 5 e^{2}\left[2-9 c+\frac{2 \beta^{2}}{25 \eta^{5}}\left(121-105 c-75 c^{2}\right.\right. \\
- & \left.\left.\left.\frac{8(2+3 \eta)}{(1+\eta)^{2}}\right)\right](1+c)^{2} \cos (2 g+2 h)\right\}
\end{aligned}
$$

$$
\begin{aligned}
& \delta \ell^{\prime \prime}=\frac{\eta}{1024}\left\{288\left[45-34 \eta^{2}-\frac{8 \beta^{2}}{5 \eta^{5}}\left(5+3 \eta^{2}-\frac{9 \beta^{2}}{\eta^{3}}\right)\right] c s^{2} \sin 2 h\right. \\
& -240\left[(2+9 c)\left(3-2 \eta^{2}\right)+\frac{\beta^{2}}{25 \eta^{5}}\left(q_{1}+15(7-5 c)\left(1+\eta^{2}\right) c\right)\right] \\
& \times(1-c)^{2} \sin (2 g-2 h)-240\left[(2-9 c)\left(3-2 \eta^{2}\right)\right. \\
& \left.+\frac{\beta^{2}}{25 \eta^{5}}\left(q_{1}-15(7+5 c)\left(1+\eta^{2}\right) c\right)\right](1+c)^{2} \sin (2 g+2 h) \\
& +90 s^{2} q_{2}\left[2\left(1+5 c^{2}\right) \sin 2 g-5(1-c)^{2} \sin (2 g-4 h)\right. \\
& \left.-5(1+c)^{2} \sin (2 g+4 h)\right]+225\left[2 s^{4} \sin 4 g\right. \\
& \left.\left.-(1-c)^{4} \sin (4 g-4 h)-(1+c)^{4} \sin (4 g+4 h)\right]\right\} \\
& q_{1}=89+121 \eta^{2}+16(1+2 \eta) /(1+\eta)^{2} \\
& q_{2}=1+(3 / 5)\left(\beta^{2} / \eta^{5}\right)\left(7-5 \eta^{2}\right)
\end{aligned}
$$

$$
\begin{aligned}
\delta g^{\prime \prime} & =\frac{3}{2048 \eta^{2}}\left\{6 0 \left[5-5 c^{4}-\left(7+20 c^{2}-15 c^{4}\right) \eta^{2}\right.\right. \\
& \left.+12 c^{2} \eta^{4}+\frac{12 \beta^{2}}{5 \eta^{3}}\left(4-10 c^{4}-\left(5+4 c^{2}-15 c^{4}\right) \eta^{2}\right)\right] \sin 2 g \\
& +192 c\left[15 c^{2} \eta^{2}-17 \eta^{4}+\frac{8 \beta^{2}}{5 \eta^{3}}\left(\frac{3 \beta^{2}}{\eta^{3}}\left(4-5 c^{2}\right)+5\left(5-7 c^{2}\right)\right.\right.
\end{aligned}
$$$$
\left.\left.-3\left(3-5 c^{2}\right) \eta^{2}\right)\right] \sin 2 h-160(1-c)\left[\left(1+c+9 c^{2}\right) \eta^{2}\right.
$$$$
-(3+8 c) \eta^{4}+\frac{\beta^{2}}{25 \eta^{3}}\left(\frac{16(1-c)}{(1+\eta)^{2}}-\frac{16(5-7 c)}{1+\eta}-356+9 c\right.
$$$$
+1185 c^{2}-600 c^{3}-24(3-5 c) \eta+\left(242-169 c-825 c^{2}\right.
$$$$
\left.\left.\left.+450 c^{3}\right) \eta^{2}\right)\right] \sin (2 g-2 h)-160(1+c)\left[\left(1-c+9 c^{2}\right) \eta^{2}\right.
$$$$
-(3-8 c) \eta^{4}+\frac{\beta^{2}}{25 \eta^{3}}\left(\frac{16(1+c)}{\left(1+\eta^{2}\right)}-\frac{16(5+7 c)}{1+\eta}-356\right.
$$$$
-9 c+1185 c^{2}+600 c^{3}-24(3+5 c) \eta+(242+169 c
$$$$
\left.\left.\left.-825 c^{2}-450 c^{3}\right) \eta^{2}\right)\right] \sin (2 g+2 h)+30(1-c)[5(1+c
$$$$
\left.+3 c^{2}-c^{3}\right)-\left(1+21 c+25 c^{2}-15 c^{3}\right) \eta^{2}+6(1+c) \eta^{4}
$$$$
-\frac{12 \beta^{2}}{5 \eta^{3}}\left(8-7 c-15 c^{2}+10 c^{3}-\left(13-12 c-20 c^{2}\right.\right.
$$$$
\left.\left.\left.+15 c^{3}\right) \eta^{2}\right)\right] \sin (2 g-4 h)+30(1+c)\left[5 \left(1-c+3 c^{2}\right.\right.
$$$$
\left.+c^{3}\right)-\left(1-21 c+25 c^{2}+15 c^{3}\right) \eta^{2}+6(1-c) \eta^{4}
$$$$
-\frac{12 \beta^{2}}{5 \eta^{3}}\left(8+7 c-15 c^{2}-10 c^{3}-\left(13+12 c-20 c^{2}\right.\right.
$$$$
\left.\left.\left.-15 c^{3}\right) \eta^{2}\right)\right] \sin (2 g+4 h)+150 s^{4}\left(1-3 \eta^{2}\right) \sin 4 g
$$$$
-24 c\left[25 c^{2}-5\left(1+5 c^{2}\right) \eta^{2}+9 \eta^{4}\right.
$$$$
\left.+\frac{24 \beta^{2}}{5 \eta^{3}}\left(\frac{6 \beta^{2}}{5 \eta^{3}}+5-3 \eta^{2}\right)\right] \sin 4 h+75(1-c)^{2}[1
$$$$
\left.+2 c-c^{2}-\left(1+6 c-3 c^{2}\right) \eta^{2}+2 \eta^{4}\right] \sin (4 g-4 h)
$$$$
+75(1+c)^{2}\left[1-2 c-c^{2}-\left(1-6 c-3 c^{2}\right) \eta^{2}\right.
$$

$\left.\left.+2 \eta^{4}\right] \sin (4 g+4 h)\right\}$ 


$$
\begin{aligned}
\delta h^{\prime \prime} & =\frac{3}{1024 \eta^{2}}\left\{360 c e^{2}\left(\eta^{2}+\frac{6 \beta^{2}}{5 \eta^{3}}\right) \sin 2 g+48\left(1-3 c^{2}\right)\right. \\
& \times\left[\left(15-17 \eta^{2}\right) \eta^{2}-\frac{16 \beta^{2}}{5 \eta^{3}}\left(5-3 \eta^{2}+\frac{3 \beta^{2}}{2 \eta^{3}}\right)\right] \sin 2 h \\
& -40(1-c) e^{2}\left[(5-27 c) \eta^{2}-\frac{2 \beta^{2}}{25 \eta^{3}}(137+465 c\right. \\
& \left.\left.-300 c^{2}-\frac{16(2+3 \eta)}{(1+\eta)^{2}}\right)\right] \sin (2 g-2 h)+40(1+c) e^{2} \\
& \times\left[(5+27 c) \eta^{2}-\frac{2 \beta^{2}}{25 \eta^{3}}\left(137-465 c-300 c^{2}\right.\right. \\
& \left.\left.-\frac{16(2+3 \eta)}{(1+\eta)^{2}}\right)\right] \sin (2 g+2 h)+30(1-c) e^{2} \\
& \times\left[\frac{6 \beta^{2}}{5 \eta^{3}}(3-7 c)+3(1+c) \eta^{2}-10 c\right] \sin (2 g-4 h) \\
& -30(1+c) e^{2}\left[\frac{6 \beta^{2}}{5 \eta^{3}}(3+7 c)+3(1-c) \eta^{2}\right. \\
& +10 c] \sin (2 g+4 h)+6\left[\frac{24 \beta^{2}}{5 \eta^{3}}\left(5-3 \eta^{2}+\frac{6 \beta^{2}}{5 \eta^{3}}\right)\left(1+c^{2}\right)\right. \\
& \left.-25\left(1-3 c^{2}\right)+20\left(1-4 c^{2}\right) \eta^{2}+9\left(1+c^{2}\right) \eta^{4}\right] \sin 4 h \\
& \left.-75(1-c)^{2} e^{4} \sin (4 g-4 h)+75(1+c)^{2} e^{4} \sin (4 g+4 h)\right\}
\end{aligned}
$$

\section{Acknowledgments}

Support from projects ESP2004-04376 and ESP-2005-07107 of the Government of Spain is recognized.

\section{References}

[1] Kozai, Y., "Motion of a Lunar Orbiter," Publications of the Astronomical Society of Japan, Vol. 15, No. 3, 1963, pp. 301-312.

[2] Scheeres, D. J., Guman, M. D., and Villac, B. F., "Stability Analysis of Planetary Satellite Orbiters: Application to the Europa Orbiter," Journal of Guidance, Control, and Dynamics, Vol. 24, No. 4, 2001, pp. 778-787.

[3] Aiello, J., "Numerical Investigation of Mapping Orbits about Jupiter's Icy Moons," 2005 Astrodynamics Specialists Conference, Lake Tahoe, CA, American Astronautical Society, Paper 2005-377, Aug. 2005.
[4] Paskowitz, M. E., and Scheeres, D. J., "Orbit Mechanics About Planetary Satellites Including Higher Order Gravity Fields,” 2005 Space Flight Mechanics Meeting, Copper Mountain, CO, American Astronautical Society, Paper 2005-190, Jan. 2005.

[5] Deprit, A., "Canonical Transformations Depending on a Small Parameter," Celestial Mechanics, Vol. 1, No. 1, 1969, pp. 12-30. doi:10.1007/BF01230629

[6] Lara, M., San-Juan, J. F., and Ferrer, S., "Secular Motion Around TriAxial, Synchronously Orbiting, Planetary Satellites: Application to Europa," Chaos: An Interdisciplinary Journal of Nonlinear Science, Vol. 15, No. 4, 2005, pp. 043101-1-043101-11. doi:10.1063/1.2038547

[7] Paskowitz, M. E., and Scheeres, D. J., "Design of Science Orbits About Planetary Satellites: Application to Europa," Journal of Guidance, Control, and Dynamics, Vol. 29, No. 5, 2006, pp. 1147-1158. doi: $10.2514 / 1.19464$

[8] San-Juan, J. F., Lara, M., and Ferrer, S., "Phase Space Structure Around Oblate Planetary Satellites," Journal of Guidance, Control, and Dynamics, Vol. 29, No. 1, 2006, pp. 113-120.

[9] Lara, M., and Ferrer, S., "Computing Long Lifetime Orbits for the Europa Observation Mission," 19th International Symposium on Space Flight Dynamics, Kanazawa, Japan, International Symposium on Space Technology and Science, Paper 2006-d-03, 411 June 2006.

[10] Lara, M., and Ferrer, S., "Computing Long Lifetime Orbits Around Natural Satellites," Discrete and Continuous Dynamical Systems, Series $S$ (to be published).

[11] Bursa, M., "Figure and Dynamic Parameters of Synchronously Orbiting Satellites in the Solar System," Bulletin of the Astronomical Institutes of Czechoslovakia, Vol. 40, No. 2, 1989, pp. 125-130.

[12] Deprit, A., and Rom, A., "The Main Problem of Artificial Satellite Theory for Small and Moderate Eccentricities," Celestial Mechanics, Vol. 2, No. 2, 1970, pp. 166-206. doi:10.1007/BF01229494

[13] Henrard, J., "Virtual Singularities in the Artificial Satellite Theory," Celestial Mechanics, Vol. 10, No. 4, 1974, pp. 437-449. doi:10.1007/BF01229120

[14] Hoots, F. R., "A Nonsingular Reformulation of the Brower Geopotential Theory," Astrodynamics Specialists Conference, Provincetown, MA,American Astronautical Society, Paper 1979-137, June 1979

[15] Gómez, G., Lara, M., Russell, Ryan, P., "A Dynamical Systems Approach to the Design of the Science Orbit Around Europa," 19th International Symposium on Space Flight Dynamics, Kanazawa, Japan, International Symposium on Space Technology and Science, Paper 2006-d-02, 4-11 June 2006.

[16] Lara, M., and Russell, Ryan, P., "On the Computation of a Science Orbit About Europa," Journal of Guidance, Control, and Dynamics, Vol. 30, No. 1,2007 , pp. 259-263. doi:10.2514/1.22493

[17] Bagenal, F., Dowling, T., and McKinnon, W. (eds.), Jupiter, The Planet, Satellites, and Magnetosphere, Cambridge Planetary Society, Cambridge, England, U.K., 2004, p. 285. 